Research Paper

\title{
Procalcitonin-Guided Antibiotic Use in Acute Exacer- bations of Idiopathic Pulmonary Fibrosis
}

\author{
Juanjuan Ding ${ }^{\circledR}$, Zhuochang Chen, Keqing Feng \\ Respiratory Department, HeNan Provincial People's Hospital, China. \\ $\triangle$ Corresponding author: Juanjuan Ding Address: Respiratory Department, HeNan Provincial People's Hospital, No 7, Weiwu Road, \\ Zhengzhou 450003, HeNan Province, China. Contact phone: 86-13663852769 Fax number: 86-371-65580098 Email: dingjuanhai@163.com.
}

(c) Ivyspring International Publisher. This is an open-access article distributed under the terms of the Creative Commons License (http://creativecommons.org/ licenses/by-nc-nd/3.0/). Reproduction is permitted for personal, noncommercial use, provided that the article is in whole, unmodified, and properly cited.

Received: 2012.08.02; Accepted: 2013.04.17; Published: 2013.05.20

\begin{abstract}
Object: To assess the clinical value of procalcitonin to guide antibiotic therapy in acute exacerbations of idiopathic pulmonary fibrosis. Methods: Patients with acute exacerbations of idiopathic pulmonary fibrosis were randomly assigned to the procalcitonin-guided group (antibiotic use guided by a procalcitonin threshold of $0.25 \mathrm{ng} / \mathrm{ml}$ ) or the routine treatment group (antibiotic use according to routine practice). Follow up of clinical outcomes were assessed at baseline and 30 days later. Results: Baseline characteristics including demographics, clinical characteristics and laboratory results were similar between groups. PCT guidance resulted in a significant reduction of antibiotic treatment duration ( $8.7 \pm 6.6$ compared to $14.2 \pm 5.2$ days in the routine treatment group). Fewer patients were exposed to antibiotics treatment in the PCT group (26 patients) compared with the control group (35 patients). Treatment success, mortality rate, days of hospitalization and ventilation therapy were similar between the two groups. Conclusion: Procalcitonin-guided antibiotic therapy of patients with acute exacerbation of idiopathic pulmonary fibrosis may result in reduced exposure to antibiotics without adversely affecting patient outcomes.
\end{abstract}

Key words: procalcitonin; antibiotic use; acute exacerbation of idiopathic pulmonary fibrosis (AE-IPF).

\section{Introduction}

Idiopathic pulmonary fibrosis (IPF) is a chronic, progressive, fatal form of diffuse interstitial lung disease which is associated with substantial mortality and a median survival of 2.5 to 5 years from the time of diagnosis ${ }^{1}$. In spite of acute exacerbations of IPF (AE-IPF) being the most common cause of death in this patient population, the pathogenesis of AE-IPF is unknown and diagnosis is reliant upon exclusion of other diseases of respiratory compromise, such as infection, pulmonary edema and embolism². Given the inherent difficulties in diagnosing pulmonary infections, many patients are treated with prolonged courses of antibiotic therapy even when a causative pathogen is not identified ${ }^{3}$. Excessive use of antibiotics is associated with increased antibiotic resistance, high costs, and adverse antibiotic reactions ${ }^{4}$. A novel approach to assess the presence of an infection and its response to treatment is the use of biomarkers ${ }^{5}$. Procalcitonin (PCT), a calcitonin precursor, is elevated in response to microbial toxins and certain bacteria-specific proinflammatory mediators. In addition, there is a strong correlation between the concentration of PCT and the extent and severity of bacterial infection ${ }^{6}$.

Recent studies have shown that PCT-guided antibiotic therapy has reduced antibiotic prescriptions and duration of antibiotic therapy in patients with COPD exacerbation and community-acquired pneumonia, accordingly ${ }^{7}$. The aim of this study was to determine if the same outcomes can be achieved with 
PCT-guided antibiotic therapy of AE-IPF.

\section{Patients and Methods}

\section{Study population}

This study was performed in HeNan Provincial People's Hospital, a 3,000-bed tertiary care hospital. Ethics commission approval was obtained from HeNan Provincial People's Hospital.

All patients with suspected AE-IPF admitted to the respiratory department were assessed for eligibility from January 2009 to December 2011. AE-IPF was defined according to the criteria established by the Idiopathic Pulmonary Fibrosis Clinical Research Network ${ }^{8}$ : (1) previous or concurrent diagnosis of idiopathic pulmonary fibrosis; (2) unexplained worsening or development of dyspnea within 30 days; (3) high-resolution computed tomography with new bilateral ground-glass; (4) abnormality and/or consolidation superimposed on a background reticular or honeycomb pattern consistent with usual interstitial pneumonia pattern; (5) no evidence of pulmonary infection by endotracheal aspirate or bronchoalveolar lavage; (6) exclusion of left heart failure, pulmonary embolism and identifiable cause of acute lung injury. Patients treated with antibiotics during the previous 2 weeks were excluded.

\section{Procedure}

Patients were randomly assigned to either PCT-guided antibiotic treatment or a control group receiving routine antibiotic therapy by the statistician using computer-generated random numbers. This process prevents investigator from knowing in advance the treatment to which subjects will be assigned. All patients signed the informed consents. Patients in both groups underwent daily routine laboratory analysis including blood cell count, arterial blood gas analysis and blood biochemical tests. In the PCT-guided group, serum PCT level was measured every three days. The first PCT measurement was available before the clinical decision to start antibiotics treatment. If the physician violated the protocol, the patient was defined as "withdrawn". Patients whose serum PCT value exceeded the threshold of $0.25 \mathrm{ng} / \mathrm{ml}$ were administered antibiotics. If antibiotics were initiated, patients were treated until PCT value fell to $\leq 0.25 \mathrm{ng} / \mathrm{ml}$. In the routine treatment group, patients were treated by antibiotics according to the clinical experience of clinicians typically guided by conventional laboratory tests, such as sputum bacteriology and white blood cell count.
Serum PCT was measured with a time-resolved amplified cryptate emission technology assay (Kryptor PCT; Brahms AG, Henningsdorf, Germany) with a functional assay sensitivity of $0.06 \mathrm{ng} / \mathrm{ml}$, about fourfold above mean normal levels ${ }^{9}$. Age, gender, length of hospitalization, the numbers of patients exposed to antibiotics treatment, duration of antibiotic treatment, cases of mechanical ventilation, outcome were retrieved from electronic medical records. The data were analyzed per-protocol analysis.

\section{Statistics Analysis}

Discrete variables are expressed as counts (percentage) and continuous variables as mean \pm SD. Comparability of the control group and the procalcitonin group was analyzed by chi-square tests, two sample t-test or Mann-Whitney $U$ test. All tests were two-tailed and P-values $\leq 0.05$ were considered statistically significant. Statistical analyses were performed by using the SPSS version 16.0 (SPSS, Inc., Chicago, IL, USA).

\section{Results}

Overall, 102 patients with AE-IPF were screened for eligibility. Of these patients, 78 patients met the inclusion criteria by fulfilling the definition of AE-IPF and were randomized. Thirty-nine patients were assigned to the PCT-guided treatment group and 39 patients to the routine treatment group. Ten patients were lost to follow up or withdrew from the study (Figure 1).

The baseline characteristics on admission were similar in both groups (Table 1). The average age of onset was 72 years in the PCT group and 73 years in the routine group $(P=0.63)$. All patients presented dyspnea and most of them had dry cough. Inspiratory crackles were heard on physical examination in most cases (26 in the PCT group vs 24 in the routine treatment group; $P=0.20$ ). The two groups were comparable with respect to gender, smoking history, duration of illness and symptoms. No difference was found with regard to blood gas analysis, white blood cell count, duration of corticosteroid and immunosuppressive therapy. As compared with the routine treatment group, the median antibiotic duration was 5.8 days shorter in the PCT group ( $P=0.0001)$, and the patients exposed to antibiotics treatment were fewer in the PCT group. Duration of mechanical ventilation was similar in patients in the PCT group and the routine group $(P=0.49)$. Mortality did not differ between the two groups $(P=0.42)$. 


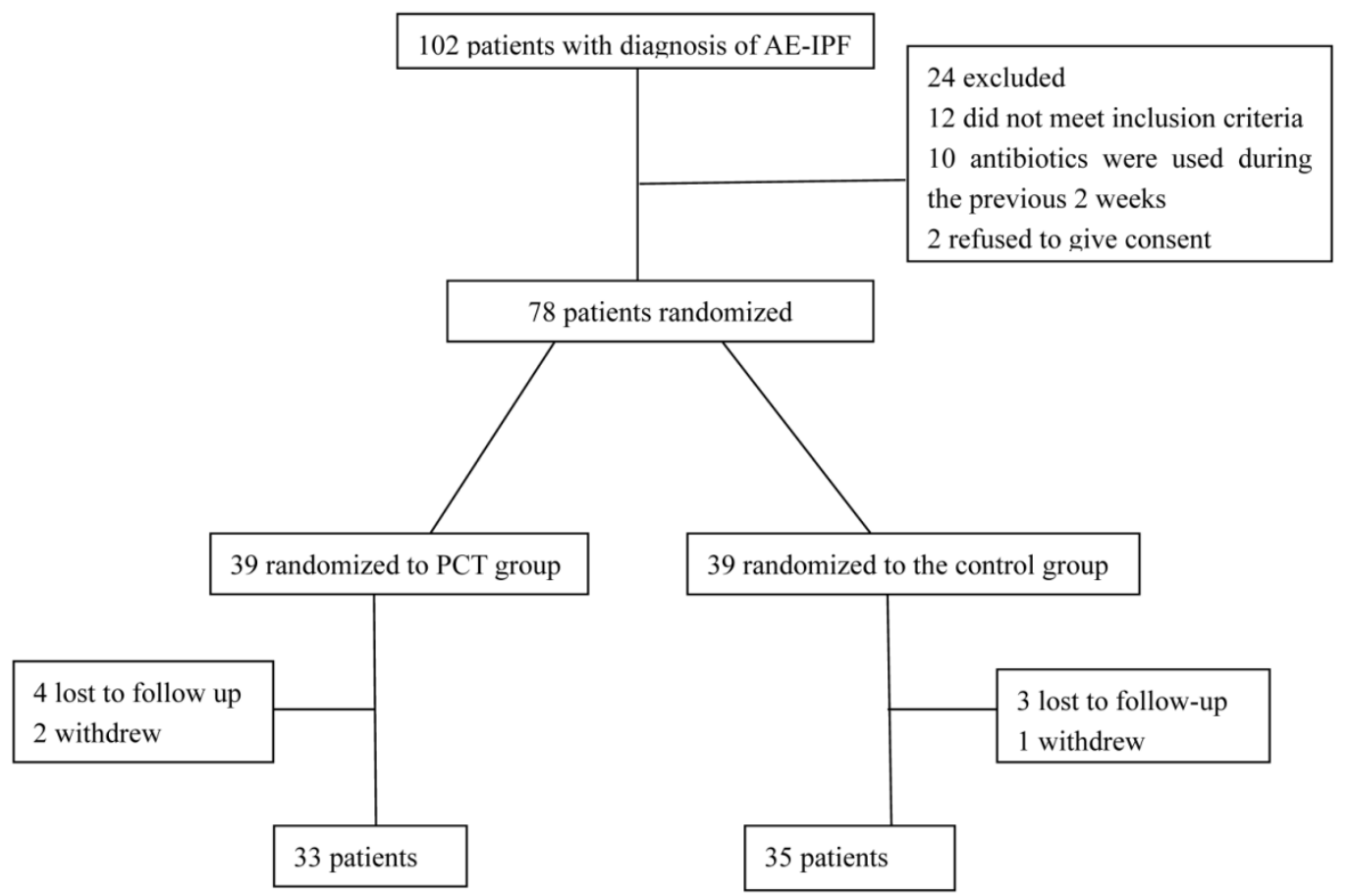

Figure I. Flowchart showing enrollment of patients with AE-IPF.

Table I. Characteristics of patients with acute exacerbation of idiopathic pulmonary fibrosis.

\begin{tabular}{llll}
\hline Characteristics & PCT Group (33 cases) & Routine treatment Group (35 cases) & $P$ value \\
\hline Age (years) & $72.3 \pm 5.5$ & $73.0 \pm 6.30$ & 0.63 \\
Male sex, n (\%) & $19(57.6)$ & $19(54.3)$ & 0.61 \\
Smoker & 20 & 26 & 0.92 \\
Duration of disease (months) & $12 \pm 8.6$ & $11.6 \pm 8.7$ & 0.85 \\
Dry cough & 23 & 24 & 0.71 \\
Clubbing & 16 & 20 & 0.30 \\
Inspiratory crackles & 26 & 24 & 0.20 \\
Fever & 8 & 13 & 0.13 \\
Duration of Corticosteroid therapy (months) & $8.6 \pm 8.7$ & $7.6 \pm 7.3$ & 0.50 \\
Duration of Immunosuppressive therapy (months) & $1.7 \pm 3.2$ & $2.1 \pm 3.4$ & 0.60 \\
PaO (mmHg) & $52.1 \pm 2.7$ & $52.0 \pm 2.8$ & 0.89 \\
WBC (×10 $)$ & $10.3 \pm 3.2$ & $9.7 \pm 3.2$ & 0.41 \\
Neut $(\%)$ & $67.9 \pm 7.7$ & $69.7 \pm 7.5$ & 0.33 \\
Patients exposed to antibiotics treatment & 26 & 35 & $<0.001$ \\
Duration of antibiotic treatment (days) & $8.7 \pm 6.6$ & $14.5 \pm 5.2$ & $<0.001$ \\
Duration of mechanical ventilation (days) & $14.3 \pm 7.4$ & $15.7 \pm 8.2$ & 0.49 \\
Mortality (\%) & $21(63.6)$ & $20(57.1)$ & 0.42 \\
\hline
\end{tabular}

"clinical outcomes at 30 day follow up in patients with AE-IPF. Definition of abbreviations: $\mathrm{WBC}=$ white blood cell; Neut $=$ neutrophil.

\section{Discussion}

This is the first study to explore the utility of PCT-guided antibiotic therapy for treatment of patients with suspected AE-IPF. We found that PCT-guided therapy significantly reduced the numbers of patients exposed to antibiotics treatment and duration of antibiotic therapy without adversely af- fecting patient outcome. During the 30-days follow-up, both the mortality and duration of ventilation were similar between the PCT guided group and the routine treatment group.

AE-IPF is an acute, clinically significant deterioration of unidentifiable cause in a patient with underlying IPF and associated with a mortality rate of $70 \%{ }^{14}$. Patients with AE-IPF are vulnerable to oppor- 
tunistic infections, since they are frequently treated by high doses of corticosteroids, immunosuppressive agents and invasive therapy. AE-IPF is difficult to differentiate from infection because both present with similar clinical symptoms and signs including: fever, dyspnea, leukocytosis, elevated C-reactive protein (CRP) and ground-glass opacities on the CT scan of chest. Sputum, blood and BAL culture often lack the sensitivity to rule out pulmonary infection. Furthermore, patients with AE-IPF are often too ill to undergo bronchoscopy with BAL or lung biopsy ${ }^{15}$. Then broad-spectrum antibiotics were also used in most cases. Recent data from the literature indicate that antibiotic treatment for more than 7 days increases the risk of fungal infections; after more than 10 days of treatment, the incidence of drug-resistant organisms is significantly rising ${ }^{10}$. It is necessary to seek a highly sensitive biomarker to provide more effective and reliable basis for the differential diagnosis and guide treatment of AE-IPF.

Inflammatory markers, such as CRP or white blood cells (WBC) count, lack specificity for bacterial infections ${ }^{16}$. Reliability of CRP for guiding antimicrobial therapy is limited by its protracted response with late peak levels and a suboptimal specificity, especially in patients with systemic inflammation ${ }^{4}$. Interestingly, CRP levels were elevated in infection and systemic inflammation, although they were much higher in infections than in AE-IPF, and CRP levels were a significant prognostic factor of $\mathrm{AE}-\mathrm{IPF}^{17}$. In these respects, PCT seems more accurate than the currently available biomarkers. To date, the concept of PCT-guided antibiotic therapy has been proven in 11 RCTs including over 3500 patients ${ }^{18}$. It has been shown that PCT guidance allows reducing the use of antibiotics in patients presenting with symptoms of lower respiratory tract infection, in patients with acute exacerbation of chronic bronchitis, and in patients with community-acquired pneumonia ${ }^{19,20}$.

There are several limitations in the present study. First, the study was a single center with a relatively small sample of patients and it was not sufficiently powered to show significant differences in mortality between the groups. While AE-IPF is clinically important, its rarity makes it difficult to study in a large number of patients. Future research should confirm these results in a larger, multicentre study. Second, the follow-up period was relatively short. However, considering the short survival of IPF, Virginie Simon-Blancal ${ }^{11}$ et al reported that death of AE-IPF occurred in the first month after admission. Third, the diagnosis of AE-IPF can be challenging. Infection is difficult to exclude because of lacking of a standardized approach to rule-out infection. Infection may not have been ruled out in all patients. Fourth, more variables should be monitored in the study, such as relapse rate, reinfection, cost etc, which are always among the relevant monitored parameters in PCT studies ${ }^{21}$.

In summary, the results of this study suggest that a PCT-guided strategy applied in AE-IPF patients reduces exposure to antibiotics and the duration of antibiotics treatment, and this strategy is not associated with worse outcomes. Future studies on larger groups and multi-center validation are needed to confirm that monitoring of PCT is a valuable tool for therapeutic decision making concerning the length of antibiotic treatment and economic factors. In China with high prescription rates, the reduction in antibiotic use with PCT-guided therapy could have a major favorable impact on bacterial resistance, health costs and risks for drug-related adverse events.

\section{Competing Interests}

The authors have declared that no competing interest exists.

\section{References}

1. American Thoracic Society, European Respiratory Society. American Thoracic Society/European Respiratory Society international multidisciplinary consensus classification of the idiopathic interstitial pneumonias. Am J Respir Crit Care Med 2002;165:277-304

2. Daniels CE, Yi ES, Ryu JH. Autopsy findings in 42 consecutive patients with idiopathic pulmonary fibrosis. Eur Respir J. 2008;32:170-174.

3. Hyzy R, Huang S, Myers J, et al. Acute exacerbation of idiopathic pulmonary fibrosis. Chest. 2007;132:1652-1658.

4. Briel M, Schuetz P, Mueller B, et al. Procalcitonin-guided antibiotic use vs a standard approach for acute respiratory tract infections in primary care. Arch Intern Med. 2008 ;168:2000-2007.

5. Christ-Crain, Stolz D, Bingisser R, et al. Procalcitonin guidance of antibiotic therapy in community-acquired pneumonia: a randomized trial. Am J Respir Crit Care Med. 2006;174:84-93.

6. Gogos CA, Drosou E, Bassaris HP, et al. Pro-versus anti-inflammatory cytokine profile in patients with severe sepsis: a marker for prognosis and future therapeutic options. J Infect Dis. 2000; 181:176-180.

7. Burkhardt $\mathrm{O}$, Ewig $\mathrm{S}$, Haagen $\mathrm{U}$, et al. Procalcitonin guidance and reduction of antibiotic use in acute respiratory tract infection. Eur Respir J. 2010;36:601-7.

8. Collard HR, Moore BB, Flaherty KR,et al. Idiopathic Plumoary Fibrosis Clinical Research Network investigators. Acute exacerbations of idiopathic pulmonary fibrosis. Am J Respir Crit Care Med. 2007; 176: 636-643.

9. Nylen ES, Muller B, Becker KL, et al. The future diagnostic role of procalcitonin levels: the need for improved sensitivity. Clin Infect Dis. 2003;36:823-824.

10. Antoniou KM, Cottin V. The challenge of acute exacerbation of pulmonary fibrosis. Respira-tion. 2012;83:13-6.

11. Schroeder S, Hochreiter M, Koehler T, et al. Procalcitonin(PCT)-guided algorithm reduces length of antibiotic treatment in surgical intensive care patients with severe sepsis: results of a prospective randomized study. Langenbecks Arch Surg. 2009; 394: 221-6.

12. Simon-Blancal V, Freynet $\mathrm{O}$, Nunes $\mathrm{H}$, et al. Acute exacerbation of idiopathic pulmonary fibro-sis: outcome and prognostic factors. Respiration. 2012;83:28-35

13. American Thoracic Society; European Respiratory Society. American Thoracic Society/European Respiratory Society International Multidisplinary Consensus Classification of the Idiopathic Interstitial Pneumonias. This joint statement of the American Thoracic Society(ATS), and the European Respiraotry Society(ERS) was adopted by the ATS board of 
directors,June 2001 and by the ERS Executive Committee, June 2001. Am J Respir Crit Care Med. 2002;165:277-304.

14. Yokoyama $\mathrm{T}$, Kondoh $\mathrm{Y}$, Taniguchi $\mathrm{H}$, et al. Noninvasive ventilation in acute exacerbation of idiopathic pulmonary fibrosis. Intern Med. 2010;49:1509-14.

15. Horita N, Akahane M, Okada Y, et al. Tacrolimus and Steroid Treatment for Acute Exacerbation of Idiopathic Pulmonary Fibrosis. Intern Med. 2011;50:189-195.

16. Robert Hyzy, Steven Huang, Jeffrey Myers, et al. Acute Exacerbation of Idiopathic Pulmonary Fibrosis. Chest. 2007;132:1652-1658.

17. Schuetz P, Albrich W, Mueller B. Procalcitonin for diagnosis of infection and guide to antibiotic decision: past, present and future. BMC Med. 2011;9:107.

18. Simon-Blancal V, Freynet $\mathrm{O}$, Nunes H, et al. Acute exacerbation of idiopathic pulmonary fibrosis: outcome and prognostic factors. Respiration. 2012;83:28-35.

19. Schuetz $P$, Albrich $W$, Christ-Crain $M$, et al. Procalcitonin for guidance of antibiotic therapy. Expert Rev Anti Infect Ther. 2010; 8:575-587.

20. Nobre V, Harbarth S, Graf JD,et al. Use of procalcitonin to shorten antibiotic treatment duration in septic patients: a randomized trial. Am J Respir Crit Care Med. 2008; 177:498-505.

21. Kopterides P, Siempos II,Tsangaris I, et al. Procalcitonin-guided algorithms of antibiotic therapy in the intensive care unit: a systematic review and meta-analysis of randomized controlled trials. Crit Care Med. 2010; 38:2229-41. 